More than $40 \%$ of the respondents selected train-thetrainer resources, guest speakers, and online courses as being helpful. However, $<30 \%$ felt that seminars were useful. These survey results validate the general assumption that nursing programs provide limited content in disaster preparedness. The mean values of the number of hours of disaster preparedness content did not change significantly over the three academic years, only approximately four hours (ranged from 3.9 in $2000-2001$ to 4.2 in 2002-2003). The study also indicated that $75 \%$ of the respondents felt that nursing faculty were prepared inadequately in the area of disaster nursing.

This study demonstrated the lack of emergency preparedness curriculum content in US nursing programs and established a baseline for future curriculum growth. In addition, there are implications for future faculty development in emergency preparedness. The study should be replicated in other countries for comparison.

Keywords: curricula; education; emergency preparedness; nurses; training; United States

Prebosp Disast Med 2005;20(2):s36-s 37

\section{Education on Emergency Preparedness for Public Health Nurses in Florida}

S.A. Straub; P.J. Gardner; K.T. Albers

Florida Department of Health, USA

The Florida Department of Health's Office of Public Health Nursing (OPHN) has developed awareness and operationallevel training to prepare public health nurses (PHN) to respond to all types of hazards/emergencies. The awareness level focuses on general understanding of preparedness concepts pertinent to job functions. The operational level focuses on the application of preparedness concepts pertinent to job functions. Both courses are based on the core competencies developed by Columbia University in cooperation with the Centers for Disease Control and Prevention (CDC).

The awareness-level course is web-based and can be taken online. Lessons learned during the 2004 hurricane season have been incorporated into the curriculum. Brief tests presented at the conclusion of each section of the course are used to assess student outcome. Continuing education credit is awarded.

A team of trainers teaches the operational-level course. Each student completes a learning needs assessment before class attendance. Information from these assessments allows for personalization of teaching methods. Didactic sessions provide information on natural, chemical, biological, radiological, nuclear, and explosive hazards and the public health nurse's role and response to such hazards/emergencies. Concepts such as incident command, triage, communication, and surveillance are included. Whenever possible, concepts are illustrated using "disasters" occurring in the world at the time of the class. Students who recently have been involved in an emergency response are encouraged to contribute information from their experience. Skill drills (table-top exercises) on selected concepts are interspersed throughout the day to encourage the application of these concepts.

Because Florida is a large state with many public health nurses, a team of trainers is being recruited to provide the operational-level course to all public health nurses. Preparation to be a trainer includes attendance at the course, as well as a "train the trainer" course, which will include topics on adult education. The trainers and course presentation will be evaluated through course evaluation documents and periodic audits of actual classes.

Keywords: awareness; education; emergency; Florida; operational; preparedness; public health nurses; response; training Prebosp Disast Med 2005;20(2):s37

\section{Standardizing Expectations of Nursing Contributions to Emergency Response: The Role of International Nursing Coalition for Mass Casualty Education (INCMCE) K. Gebbie, ${ }^{1}$ C. Conway-Welsh, ${ }^{2}$ R. Lavin, ${ }^{3}$ M.P. Couig; ${ }^{4}$ J. Stanley; ${ }^{5} M$. Chaffee $e^{6}$ \\ 1. Columbia University School of Nursing, USA \\ 2. Vanderbilt University, USA \\ 3. United States Department of Health and Human Services, USA \\ 4. Food and Drug Administration, Department of Heath and Human Services, USA \\ 5. American Association of Colleges of Nursing, USA \\ 6. Department of Defense, USA}

The International Nursing Coalition for Mass Casualty Education (INCMCE) was formed in 2001 to increase the assurance that anyone developing a response to emergencies (including mass-casualty events, bioterrorism, or large natural disasters) could rely on nurse responders to have a common and appropriate ability to fit into an incident management system, fulfill typical functional roles requiring nursing skills, and do so in an ethical manner. The development of the required compentencies has involved nurses from five nations already active in their own national emergency improvement programs, and (in the United States) nurses from both uniformed services and civilian organizations.

The INCMCE Competencies for Nurses were published in 2003, and have been widely distributed in both electronic and hard copy form. The INCMCE has moved to develop action plans for the incorporation of the competencies in nursing education, and for the development of an agenda of nursing research that would facilitate both improved education and improved emergency nursing response.

Keywords: competencies; emergency; nursing; response Prehosp Disast Med 2005;20(2):s37

\section{An Online Emergency Curriculum for Nurses Internationally \\ E. Weiner, ${ }^{1}$ M. Irwin; ${ }^{1}$ S. Woods; ${ }^{2}$ L. Norman ${ }^{1}$ \\ 1. Vanderbilt University, USA \\ 2. Little Planet Learning, Inc., USA}

The Vanderbilt University School of Nursing has taken a leadership role with the establishment of the International Nursing Coalition for Mass-Casualty Education (INCM$\mathrm{CE}$ ). This coalition consists of organizational representatives of schools, accrediting bodies, specialty organizations, and governmental agencies interested in promoting masscasualty education for nurses. Several grants have been obtained to develop an online curriculum that addresses the competencies as developed by the INCMCE members for all nurses. 
The objectives of this presentation are to: (1) familiarize participants with the INCMCE; and (2) demonstrate a representative module of the INCMCE curriculum.

The first completed module, called "The Tipping Point", is designed to help nurses identify possible changes in care that occur when mass casualties are received, describe differing triage principles, and discuss the psychological support needed during and after a mass-casualty event. The modules have been designed using the "How People Learn" framework, a format that was developed after a review of the educational literature sponsored by the National Research Council (USA).

Four of the six proposed modules are in development. A complete set of modules will provide nurses with the resources needed to meet the international competencies.

Drawing on the experiences of nurses internationally allows for a wealth of testimonials and resources from which all can learn in an online format interactively.

Keywords: education; emergency; international; International Nursing Coalition for Mass-Casualty Education (INCMCE); modules; nurses; online

Prebosp Disast Med 2005;20(2): :37-s38

\section{Preparing Volunteer Nurses for Public Health Emergencies}

E. Weiner; P. Trangenstein; M. Irwin; L. Norman;

$J$. Gordon

Vanderbilt University, Nashville, Tennessee USA

Several grants have been received to develop an online curriculum for nurses in emergency preparedness using the competencies developed by the International Nursing Coalition for Mass-Casualty Education (INCMCE).

This presentation will provide data to determine the effectiveness and efficiency of learning programs designed to educate nurses volunteering for service in their local community Medical Reserve Corps (MRC) or statewide volunteer nurses. Two types of learning programs will be compared: (1) a face-to-face version; and (2) an online version, both of which will be designed using the principles of the national How People Learn (HPL) framework.

Effectiveness of the learning programs will be determined by: (1) mastering course content; (2) following International Nursing Coalition for Mass Casualty (INCMCE) competencies; (3) incorporating principles of the HPL framework; (4) promoting course completion; and (5) increasing clinical confidence. Efficiency will be measured by cost per student, time spent in completion, and convenience for the student.

Additional aims of the project will be to define user characteristics that predict selection of and effective/efficient completion of learning programs, and to determine the adequacy of technology integration in learning emergency response content. The long-term objective of this study is to provide quality educational materials for nurses volunteering in their local and regional communities, thereby improving the quality of health following emergency public health events in the United States.

Keywords: assessment; cmergency preparedness; management; nurses; training

Prehosp Disast Med 2005:20(2):s38

\section{Free Papers Theme 12: Tsunami-1}

\section{Free Papers Theme 13: Disaster Planning -1}

\section{Theme 9: Infectious Diseases and Epidemics \\ Chair: Frederick M. Burkle, Jr., MD, MPH}

Caring About Healthcare Workers as First Responders: Building Research Capacity to Enhance Resiliency through Emergency Preparedness Planning C. Amaratunga, ${ }^{1}$ T. O'Sullivan, ${ }^{2}$ M. Félix-Bortolotti, ${ }^{2}$ W. Corneil, ${ }^{2}$ K. Pbillips, ${ }^{2}$ R. Clarke, ${ }^{2}$ E. O'Connor; ${ }^{2}$ H. Smith-Fowler; ${ }^{2}$ L. Lemyre ${ }^{2}$

1. Institute of Population Health, Canada

2. University of Ottawa, Canada

Traditionally, healthcare workers ( $\mathrm{HCWs}$ ) have not been included as first responders in chemical, biological, radiological, and nuclear (CBRN) response policy and training. Recent terrorism and natural disasters have highlighted the need to include $\mathrm{HCW}_{\mathrm{s}}$ in CBRN emergency preparedness plans. Using the severe acute respiratory syndrome (SARS) outbreaks as a model, this four-year study, funded by the CBRN Research Technology Initiative, focuses on frontline HCW response, preparedness, and resilience for coping with disaster scenarios.

The review of existing literature in three distinct areas includes: (1) support mechanisms for $\mathrm{HCW}$ as first responders; (2) impacts on HCWs involved in SARS and other outbreaks; and (3) mechanisms for adoption of the best practices of communication and organization. A survey is being conducted currently of civilian and military HCWs focusing on family and health impacts of firstresponder roles. In conjunction with the survey data, a third component of the study entails identification, risk assessment, and analysis of hospital employee support mechanisms during an outbreak. Evaluations of emergency plans for three hospitals, and focus group data from emergency team members, are being used to identify gaps in support mechanisms for front-line HCWs and develop policy recommendations for a risk management framework for public health emergency preparedness. Additional analyses examine personnel policy and work-family conflict from a gender perspective.

It is clear that the SARS outbreak in Canada has provided a stimulus for increased attention to $\mathrm{HCW}$ s as first responders in infectious disease outbreaks, however communications and policy recommendations are needed to ensure these groups are supported in their roles.

Keywords: Canada; chemical, biological, radiological, nuclear (CBRN); disasters; healthcare workers $\left(\mathrm{HCW}_{\mathrm{s}}\right)$; infectious diseases; outbreaks; preparedness; response; terrorism Prebosp Disast Med 2005;20(2):s38 